\section{Just a light squeeze}

\section{R. S. Pease}

LASER radiation has been used to compress matter to a record density in experiments detailed at a recent conference* on plasma physics. Part of the drive to generate nuclear fusion using inertial confinement', the experiments (S. Nakai, Osaka Univ.) used 8-10 kilojoule pulses of focused, short-wavelength laser light to compress small carbon-deuteriumtritium capsules to a density of 600 tons per cubic metre, about three times that reported from other laboratories ( $R$. McCrory, Rochester Univ.).

Expressed in terms of the number of electrons per unit volume, the peak density at $10^{32} \mathrm{~m}^{-3}$ reported from Osaka is comparable to that in our nearest natural fusion reactor, the Sun's core. The measured temperature, on the other hand, is about a fifth that in the Sun's core. One consequence is that the characteristic 'Fermi' energy of such compressed plasma (about $1,000 \mathrm{eV}$ ) exceeds the thermal energy $(300 \mathrm{eV})$, so that a regime in plasma physics new to the laboratory, in which the electrons are degenerate most in the lowest energy states - may be created.

The measured densities, obtained by monitoring the number of secondary reactions of the fusion products with heavier nuclei present in the laser pellets (which depends on the density and radius) and an estimate of the mass of matter compressed, are accurate to within a factor of two or so. Nevertheless, they agree with that calculated on the assumption that the laser pulses implode the pellets spherically symmetrically. But the fusion neutron yields tell a different story. For radial compression ratios more than about 20 , the yield is lower than that calculated by three orders of magnitude at the highest compression. This corresponds (roughly) to the ion temperature being three times lower than expected. It could be that non-uniformity in the implosion results in some cold material being mixed into the innermost hot spark (Nakai).

The compression achievable by these techniques is limited by the RayleighTaylor instability, first investigated by Lord Rayleigh (Scientific Papers Vol. 2, 200, Cambridge Univ. Press; 1900), who was interested in the structure of cirrhus clouds in the upper atmosphere. The problem is that the inner imploding region, accelerating inwards at around $10^{-1}$ $\mathrm{m} \mathrm{s}^{-2}$, is more dense than the laser-heated outer layer that drives the implosion. Rayleigh's studies show that in these circumstances, any imperfection will quickly grow, the growth rate for an acceleration $g$

*13th IAEA Conference on Plasma Physics and Controlled Nuclear Fusion Research, Washington DC, 1-6 October 1990. being given as $\sqrt{2 \pi g / L}$, where $L$ is the wavelength of the initial imperfection when the boundary between the two layers is sharp, or the density length scale of the boundary when it is diffuse (whichever is the longer dominates). Although the use of time-varying laser intensities multiply layered targets and so on allows the designer to lessen the instabilities to a degree, the Rayleigh-Taylor instability can also occur at the centre of the pellet where imploding material is brought to a halt by a less dense, hot plasma. The potentially infinite growth rate that results from the approach of $L$ to zero on these scales is avoided in ordinary fluids by the intervention of surface tension.

But surface tension seems to have little direct effect on the imploding plasma $(\mathrm{H}$. Hora et al. Trans. Plasma Sci. IEEE 17. 284: 1989) and instead the instabilities may be stabilized by kinetic effects in the ablating outer layer. Computer calculations suggest that pertubations with wavelengths less than about $100 \mu \mathrm{m}$ (depending on the velocity of the ablated ions) might be stabilized in this way ( $\mathrm{H}$. Takabe, Osaka Univ.) and that growth rates at longer wavelengths are reduced. Experiments at Lawrence-Livermore indicate that the growth of 100$\mu \mathrm{m}$-wavelength disturbances is reduced by around a factor of two (E. Storm). A UK group, using the RutherfordAppleton Laboratory's central laser facility, report (M. Desselberger et al. Phys. Rev. Lett. 65, 2977-3000; 1990) Rayleigh-Taylor instabilities growing at $L$ values of $30 \mathrm{um}$. They did not show that ablation stabilization occurred.

Thus much of the current effort in research into inertial-confinement fusion is in minimizing the initial imperfections and in mitigating the Rayleigh-Taylor growth rates rather than eliminating them. The advance reported by the Osaka group, for example, depends on a new method for manufacturing uniform, spherically symmetric targets, the carbon-deuterium-tritium plastic shells being produced to a thickness uniform to 1 per cent. And it is now routine to use special optics for the irradiating laser beams, taylored to give a uniform distribution of intensity. Although the principal aim is to develop fusion reactors, perhaps the greatest interest in the new results lies in the prospect of studying the physics of the densest states of matter in the laboratory.

R. S. Pease, formerly Fusion Programme Director for the UK Atomic Energy Authority, is at The Poplars, West IIsley, Newbury RG16 OAW, UK
SOONER or later, the new ceramic hightemperature superconductors should find their way into power cables, which could then carry thousands of amps per square millimetre. Daedalus now points out a novel consequence of stringing such a current-dense cable across the landscape. If it carried direct current eastwards, the simple motor force of the Earth's magnetic field could levitate the cable.

At first sight, this seems a splendid way of stringing a power line between two points without expensive supporting pylons. Hundreds or even thousands of kilometres might be spanned by one magnetically levitated loop reaching far up into the ionosphere. Indeed, this is an ideal way of supporting and powering TV relay stations, space telescopes, Earth-scan cameras, and so on. But Daedalus is taking the idea to its logical conclusion. He is inventing a 'space halo' - a complete loop of cable, carrying an endlessly flowing superconductive current, magnetically levitated right around the Earth above the Equator.

The Earth's magnetic field decreases with height. So will its lift on the halo, which will therefore reach a stable equilibrium altitude. The latitudinal stability of the halo is not so obvious. A set of currentsharing subloops deviating north and south may be needed to keep it safely equatorial. Such a divided, braid-like structure would still be kept taut and expanded by the strong self-repulsion of its ring current.

This bold experiment is clearly worth trying. But simply laying 40,000 kilometres of superconducting cable loosely round the Equator and starting a current in it, is obviously impractical. Daedalus proposes instead to lay it in a meandering path around the Antarctic ice cap. (The polar cold would simplify refrigeration of the superconductor, too.) As the current was built up, the meanders would levitate to form a ring of arches. Adjacent arches would be merged by releasing the clamps between them, and more current fed in, till the cable was held down at only two or three points, arching between them up and out far into space. On releasing the final clamps, the halo would expand under its self-repulsion and swing through space to its equilibrium equatorial position.

A space-halo should be most useful at an 'auroral' height of $50-100 \mathrm{~km}$, too high for aircraft and too low for satellites. The tenuous atmosphere up there would slowly damp its initial oscillations and drag it gradually up to equatorial geosynchronous speed. Yet in such thin air, simple radiation shields to protect it from sunlight and earthlight while exposing it to the cold of space in other directions, could keep it safely cold and superconducting. It would stay up indefinitely. 